\title{
Hábitos e hipertensão: estudo realizado em hipertensos dos serviços públicos de saúde em Feira de Santana-BA
}

\author{
Habits and hypertension: study carried out on hypertensive people in public health services in \\ Feira de Santana-BA
}

\author{
Silas Santos Carvalho ${ }^{\mathrm{a}}$ \\ Orcid: http://orcid.org/0000-0001-8845-8902
}

Bruno Rodrigues de Oliveira ${ }^{b}$

Orcid: http://orcid.org/0000-0002-9693-0311

\begin{abstract}
Resumo
Introdução: Atualmente a hipertensão é um dos maiores problemas de saúde pública, reconhecida como grave fator de risco para as doenças cardiovasculares e corresponsável importante para as causas de óbito. Novas abordagens e produção de conhecimentos adicionais fazem-se oportunos e necessários, de modo a se obter maior controle dessa doença de impacto no campo da saúde pública. Objetivo: Descrever os hábitos de indivíduos hipertensos atendidos em serviços públicos de saúde em Feira de Santana-BA. Materiais e Métodos: Estudo caso-controle conduzido em indivíduos com idade mínima de 18 anos, atendidos em serviços de saúde pública no município de Feira de Santana, Bahia. O grupo caso foi formado pelos indivíduos com hipertensão, e os controles, por aqueles sem a doença. A coleta de dados foi realizada através de aplicação de questionário estruturado, consulta de prontuários e exame físico. A análise descritiva das variáveis de interesse empregou o teste Qui-quadrado de Pearson e teste T de Student, com significância de 5\%. Resultados: Obteve-se uma amostra de 870 indivíduos ( 585 casos e 285 controles), com média de idade de 52,8 anos, a maioria dos hipertensos era do sexo masculino, com mais de 4 anos de estudo, residentes na zona urbana, não praticantes de atividade física, não fumantes e não etilistas. Conclusão: $\mathrm{A}$ aplicação da mudança do hábitos de vida em indivíduos hipertensos tem efeito significativo e é indispensável, pois contribui com ações de intervenção efetivas na prevenção e tratamento do público atendido em unidades básicas de saúde.
\end{abstract}

Palavras-chave: hipertensão; perfil de saúde; hábitos de vida; epidemiologia.

\begin{abstract}
Introduction: Currently hypertension is one of the major public health problems, recognized as a serious risk factor for cardiovascular diseases and a major co-responsible for the causes of death. New approaches and the production of additional knowledge are timely and necessary, in order to obtain greater control of this disease of impact in the field of public health. Objective: Describe the habits of hypertensive individuals seen in public health services in Feira de Santana-Ba. Materials and Methods: Case control study conducted in a minimum recall of 18 years, attended at public health services without municipality of Feira de Santana, Bahia. The case group was formatted with hypertension, and controls, by those without the disease. Data collection was performed by applying a structured questionnaire, consulting medical records and physical examination. Descriptive analysis of the variables of interest employed the Pearson Chi-square test and Student's t-test, with significance of 5\%. Results: A sample of 870 individuals (585 cases and 285 controls) was obtained, with a mean age of 52,8
\end{abstract}

\footnotetext{
a Mestre em Saúde Coletiva e Enfermeiro obstétrico. Universidade Estadual de Feira de Santana (UEFS)-BA, Brasil. E-mail: ssc.academico@hotmail.com

b Universidade Salvador, Bahia. Brasil. E-mail: bruno.013@gmail.com
} 
years. Most of the hypertensive patients were male, with more than 4 years of study, living in the urban area. non-practitioners, non-smokers and non-alcoholics. Conclusion: The application of lifestyle change in hypertensive individuals has a significant effect and is indispensable, as it contributes to effective intervention actions in the prevention and treatment of the public attended at basic health units.

Keywords: hypertension; health profile; life habits; epidemiology.

\section{Introdução}

A hipertensão arterial (HA) é uma condição clínica caracterizada por níveis elevados da pressão arterial, frequentemente associada a alterações de órgãos-alvo. Atualmente, essa doença é um dos maiores problemas de saúde pública, reconhecida como grave fator de risco para as doenças cardiovasculares, além de ser uma corresponsável importante para as causas de óbito ${ }^{1-4}$.

A prevalência da HA tem sido investigada em diferentes populações e a sua ocorrência, embora alta, apresenta variações de $5 \%$ a $47 \%$, entre os homens, e $7 \%$ a $38 \%$, entre as mulheres. Tanto em países desenvolvidos quanto nos países não desenvolvidos, essa prevalência tem variado entre $30 \%$ a $45 \%$.

Dados referentes à prevalência da HA no Brasil apontam que essa doença atinge $20 \%$ a $30 \%$ em indivíduos com idade entre 18 e 59 anos, $50 \%$ na faixa etária de 60 a 69 anos e $75 \%$ em indivíduos com idade acima de 70 anos, evidenciando aumento da prevalência com a idade. ${ }^{6}$ Até o final do ano de 2015, estavam cadastrados no Sistema de Informação da Atenção Básica (SIAB), do Ministério da Saúde (MS), 6.992 .098 brasileiros hipertensos. ${ }^{7}$ Em recente pesquisa da Vigilância de Fatores de Risco e Proteção para Doenças Crônicas por Inquérito Telefônico VIGITEL $^{7}$ houve crescimento de $14,2 \%$ na última década, sendo as mulheres mais acometidas pela doença do que os homens $(27,5 \% \text { versus } 23,6 \%)^{7}$

Em Feira de Santana, segundo o $\mathrm{SIAB}^{8}$, no ano de 2015 havia 33.952 hipertensos cadastrados no município, ou seja, 54,5 casos de HA para cada mil habitantes, no entanto apenas $79 \%$ desses eram acompanhados.

Quanto aos fatores associados à HA, além da idade avançada e sexo feminino mencionados anteriormente, destacam-se outros como: obesidade, consumo de bebida alcoólica, tabagismo, sedentarismo e colesterol HDL ${ }^{9-11}$ A baixa condição socioeconômica também tem sido referida como um desses fatores, e, segundo os dados da VIGITEL, a prevalência da HA é inversamente proporcional ao nível de escolaridade $^{7}$.

Assim, justifica-se a realização deste estudo, pois são oportunas e necessárias novas abordagens e produção de conhecimentos adicionais que contribuam para ampliar a compreensão da HA e agravos sistêmicos, de modo a se obter maior controle dessa doença de impacto no campo da saúde pública. Desse modo, o presente estudo tem como objetivo descrever os hábitos de indivíduos hipertensos atendidos em serviços públicos de saúde em Feira de Santana-Ba.

\section{Material e Métodos}

Trata-se de estudo retrospectivo, analítico, do tipo caso-controle, realizado em Unidades de Saúde da Família (USF), Unidades Básicas de Saúde (UBS) da zona urbana e no Centro de Atendimento ao Diabético e Hipertenso (CADH) do município de Feira de Santana-BA, no período de junho de 2016 a janeiro de 2018.

Participaram do estudo 870 indivíduos de ambos os sexos, com idade mínima de 18 anos e que possuíam em seu cadastro/prontuário os exames laboratoriais de triglicerídeos, colesterol HDL e glicemia 
de jejum, realizados nos 30 dias que antecederam o exame clínico geral do participante e que aceitaram participar da pesquisa, mediante assinatura do termo de consentimento. Não foram selecionados para este estudo indivíduos que apresentaram uma ou mais das seguintes condições: neoplasias; gestantes e impossibilitados de comunicação verbal. $\mathrm{O}$ grupo caso foi composto pelos indivíduos com HA e o grupo controle por aqueles sem a referida condição de saúde.

Foram aplicados os questionários que englobavam dados acerca da identificação do participante, dados sociodemográficos, condição geral de saúde e hábitos de vida e, posteriormente, foi procedido o exame físico. Dados complementares foram coletados mediante solicitação de exames laboratoriais realizados em laboratório colaborador do grupo de pesquisa e consulta de prontuários.

Este estudo empregou o critério de diagnóstico padronizado pela SBC (2010) que considera hipertenso o indivíduo com Pressão Arterial Sistólica (PAS) $\geq$ $140 \mathrm{mmHg}$ ou Pressão Arterial Diastólica (PAD) $\geq 90 \mathrm{mmHg}$, ou quando o indivíduo estiver em uso de anti-hipertensivos mesmo com pressão arterial normal $\left(<120 \times 80 \mathrm{mmHg}^{6}{ }^{6} \quad \mathrm{O}\right.$ exame físico contemplou aferição dos níveis de PA, mensurações da circunferência abdominal, peso corporal e altura por um único examinador treinado e que desconhecia sobre o diagnóstico de HA do participante. $\mathrm{O}$ indivíduo foi examinado quando em repouso, com bexiga vazia e sem ter ingerido bebida alcoólica nem qualquer outro líquido nos 30 minutos anteriores à aferição. O participante foi ainda orientado para manter as pernas descruzadas, pés apoiados no chão, dorso do tronco recostado na cadeira e relaxado. ${ }^{6} \mathrm{O}$ IMC foi obtido com a razão entre o peso $(\mathrm{kg})$ e o quadrado da altura $\left(\mathrm{m}^{2}\right)$. Todas as medidas foram aferidas pelos pesquisadores treinados.

Foi realizada a análise descritiva da população quanto aos aspectos sociodemográficos, hábitos de vida, biologia humana, cuidados com a saúde e, posteriormente, foram calculadas as frequências simples e relativa das variáveis categóricas de interesse, bem como medidas de tendência central e de dispersão para as contínuas. $\mathrm{Na}$ análise bivariada foi aplicado o Teste $x^{2}$ (Qui-Quadrado) de Pearson e o Teste $\mathrm{T}$ para amostras independentes, como medidas de associação. Foi considerado um nível de significância de $5 \%(\mathrm{p}<0,05)$ e calculadas as razões de prevalência (RP) e os intervalos de confiança (IC) de $95 \%$. Os dados foram tabulados no programa Statistical Package for Social Science (SPSS) 20.0 e analisados no programa estatístico STATA versão 11.0.

Outras variáveis como escolaridade, última consulta ao dentista, colesterol HDL, obesidade e hemoglobina glicada foram incorporadas aos modelos finais (Odds ratio - OR - bruto e ajustado) a fim de se neutralizar os seus potenciais efeitos, conforme discutido na literatura.

$\mathrm{O}$ projeto foi encaminhado $\mathrm{e}$ aprovado pelo Comitê de Ética em Pesquisa (CEP) da Universidade Estadual de Feira de Santana (CAAE n ${ }^{\mathrm{o}}$ 73485317.0.0000.0053, Parecer: 2.355.697).

\section{Resultados}

Uma amostra de 870 indivíduos compôs o presente estudo. Destes, 585 $(67,2 \%)$ tiverem o diagnóstico de HA, caracterizando o grupo caso, e $285(32,8 \%)$ integraram o grupo controle. Do total, observou-se $586(67,4 \%)$ homens e 284 $(32,6 \%)$ mulheres, com média de idade de $52,8$ anos ( $\pm 14,8$ anos $)$, mínima de 18 e máxima de 89 anos. 
Tabela 1 - Características e hábitos de vida da amostra, segundo os grupos casos (hipertensos) e controles (não hipertensos) da amostra (n=870). Feira de Santana, Bahia, Brasil, 2018. Variável

Hipertensão

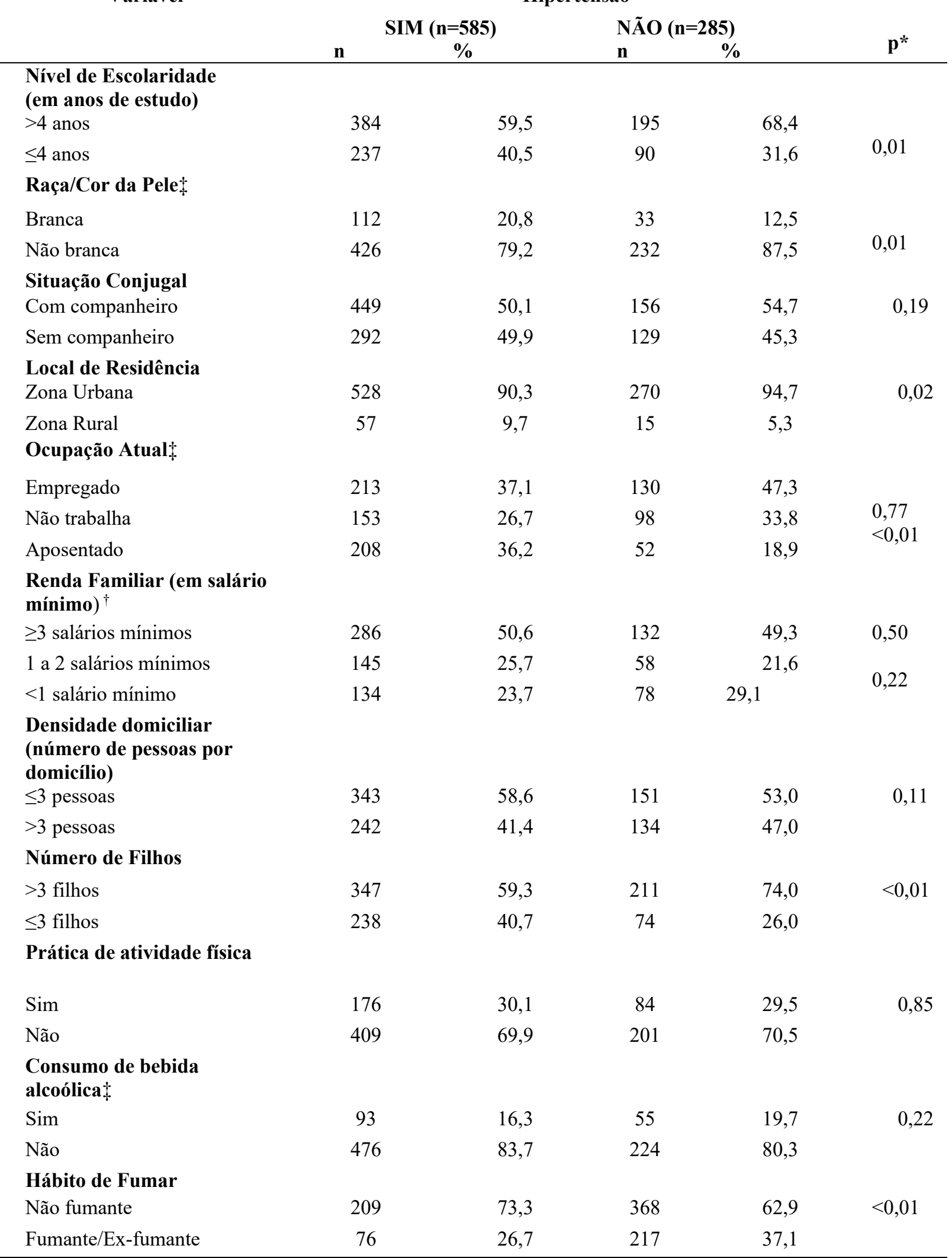

* Nível de significância estatística: $\mathrm{p} \leq 0,05$. Teste ${ }_{\mathrm{x}}^{2}$ de Pearson e Teste T;

†Valor do salário mínimo: $\mathrm{R} \$ 954,00$, na data da coleta dos dados;

†Informações perdidas ou não declaradas. 
Ao analisar as características socioeconômico-demográficas e de hábitos de vida segundo a ocorrência de HA (Tabela 1), diferenças estatisticamente significantes foram encontradas para as covariáveis: idade $(p=0,01)$, nível de escolaridade $(p=0,02)$, raça/cor da pele $(p<0,01)$, local de residência $(\mathrm{p}=0,02)$, ocupação atual $(\mathrm{p}<0,01)$, número de fillhos $(\mathrm{p}<0,01)$ e hábito de fumar $(\mathrm{p}<0,01)$. Os casos de HA, quando comparados aos controles, apresentaram maior média de idade e maiores frequências de indivíduos com até
4 anos de estudo, brancos, aposentados, com até três filhos e não fumantes.

Quanto às características relacionadas com as condições de saúde geral e dados laboratoriais segundo a ocorrência de HA (Tabela 2), observou-se que entre os casos houve maior ocorrência de pré-diabéticos e indivíduos com glicemia $\geq 126 \mathrm{mg} / \mathrm{dL} \quad(\mathrm{p}<0,01)$, hemoglobina glicada $\quad>6,5 \% \quad(p<0,01), \quad$ obesidade abdominal $\quad(p=0,01)$ e triglicérides aumentados $(\mathrm{p}<0,01)$.

Tabela 2 - Condições de saúde geral e exames bioquímicos segundo os grupos casos (hipertensos) e controles (não hipertensos) da amostra (n=870). Feira de Santana, Bahia, Brasil, 2018.

\begin{tabular}{|c|c|c|c|c|c|}
\hline \multirow[t]{3}{*}{ Características } & \multicolumn{4}{|c|}{ Hipertensão } & \multirow[b]{3}{*}{$\mathbf{p}^{*}$} \\
\hline & \multicolumn{2}{|c|}{$\operatorname{SIM}(n=585)$} & \multicolumn{2}{|c|}{ NÃO $(\mathbf{n}=\mathbf{2 8 5})$} & \\
\hline & $\mathbf{n}$ & $\%$ & $\mathbf{n}$ & $\%$ & \\
\hline \multicolumn{6}{|l|}{ Doença Cardiovascular: } \\
\hline Sim & 62 & 11,1 & 20 & 7,2 & \multirow[b]{2}{*}{0,07} \\
\hline Não & 499 & 88,9 & 258 & 92,8 & \\
\hline \multicolumn{6}{|l|}{ Doença Hepáticał } \\
\hline Sim & 23 & 4,1 & 03 & 1,1 & \multirow[t]{2}{*}{0,01} \\
\hline Não & 542 & 95,9 & 277 & 98,9 & \\
\hline \multicolumn{6}{|l|}{ Doença Pulmonarł } \\
\hline $\operatorname{Sim}$ & 06 & 1,2 & 03 & 1,3 & \multirow[t]{2}{*}{0,86} \\
\hline Não & 515 & 98,8 & 229 & 98,7 & \\
\hline \multicolumn{6}{|l|}{ Doença Renalł } \\
\hline Sim & 30 & 5,3 & 13 & 4,6 & \multirow[t]{2}{*}{0,67} \\
\hline Não & 537 & 94,7 & 269 & 95,4 & \\
\hline \multicolumn{6}{|l|}{$\operatorname{IMC}\left(\mathrm{kg} / \mathrm{m}^{2}\right)+$} \\
\hline$<25$ & 294 & 50,3 & 139 & 49,3 & \multirow{3}{*}{$\begin{array}{l}0,42 \\
0,61\end{array}$} \\
\hline$\geq 25 \mathrm{a}<30$ & 183 & 31,3 & 98 & 34,8 & \\
\hline$\geq 30$ & 108 & 18,5 & 45 & 16,0 & \\
\hline \multicolumn{6}{|l|}{ Glicemia $(\mathrm{mg} / \mathrm{dL}) \ddagger$} \\
\hline$<100$ (normal) & 127 & 22,0 & 114 & 40,6 & \multirow{3}{*}{$\begin{array}{l}<0,01 \\
<0,01\end{array}$} \\
\hline$\geq 100$ a $<126$ (pré-diabetes) & 175 & 30,3 & 69 & 24,6 & \\
\hline$\geq 126$ (diabetes) & 276 & 47,8 & 98 & 34,9 & \\
\hline \multicolumn{6}{|l|}{ Hemoglobina Glicada 6,5\% } \\
\hline$<6,5$ & 142 & 62,3 & 141 & 68,8 & \multirow[t]{2}{*}{$<0,01$} \\
\hline$\geq 6,5$ & 86 & 37,7 & 64 & 31,2 & \\
\hline \multicolumn{6}{|l|}{$\begin{array}{l}\text { Obesidade Abdominal } \$ \\
\text { ( } \geq 102 \text { em homens; } \\
\geq 88 \text { em mulheres) }\end{array}$} \\
\hline$<102$ ou $<88$ & 288 & 49,3 & 167 & 59,0 & \multirow[t]{2}{*}{0,01} \\
\hline$\geq 102$ ou $\geq 88$ & 296 & 50,7 & 116 & 41,0 & \\
\hline \multicolumn{6}{|l|}{ Triglicerídeos $(\mathrm{mg} / \mathrm{dL}) \$$} \\
\hline$<150$ & 358 & 61,7 & 211 & 74,3 & \multirow[t]{2}{*}{$<0,01$} \\
\hline$\geq 150$ & 222 & 38,3 & 79 & 25,7 & \\
\hline
\end{tabular}




\begin{tabular}{|c|c|c|c|c|c|}
\hline \multirow[t]{3}{*}{ Características } & \multicolumn{4}{|c|}{ Hipertensão } & \multirow{3}{*}{$\mathbf{p}^{*}$} \\
\hline & \multicolumn{2}{|c|}{$\operatorname{SIM}(\mathbf{n}=\mathbf{5 8 5})$} & \multicolumn{2}{|c|}{ NÃO (n=285) } & \\
\hline & $\mathrm{n}$ & $\%$ & $\mathrm{n}$ & $\%$ & \\
\hline \multicolumn{6}{|l|}{$\begin{array}{l}\text { Colesterol HDL }(\mathrm{mg} / \mathrm{dL}) \\
(<40 \mathrm{mg} / \mathrm{dL} \text { homens; } \\
<50 \mathrm{mg} / \mathrm{dL} \text { mulheres })\end{array}$} \\
\hline$<40$ ou $<50$ & 203 & 34,9 & 92 & 32,4 & 0,45 \\
\hline$\geq 40$ ou $\geq 50$ & 378 & 65,1 & 192 & 67,6 & \\
\hline \multicolumn{6}{|c|}{ Última consulta com o dentista } \\
\hline$\leq 1$ ano & 253 & 45,8 & 154 & 55,8 & $\mathbf{0 , 0 1}$ \\
\hline$>1$ ano & 300 & 54,2 & 122 & 44,2 & \\
\hline
\end{tabular}

* Nível de significância estatística: $\mathrm{p} \leq 0,05$. Teste $\mathrm{x}^{2}$ de Pearson e Teste T;

† Informações perdidas ou não declaradas.

$\mathrm{Na}$ análise multivariada, foram verificados os potenciais confundidores e modificadores dos hábitos de vida e HAS. A idade se confirmou como uma variável de interação e os modelos finais da regressão logística incluíram como covariáveis confundidoras sexo, nível de escolaridade, hábito de fumar, consumo de bebida alcóolica, colesterol HDL, consulta com dentista, DM e obesidade. As medidas brutas de associação foram estatisticamente significantes. De acordo com a Tabela 3, a chance de ter HAS foi duas vezes maior entre aqueles com hábitos de vida comprometidos (OR bruta= 2,08; IC95\%: 1,32-3,27). Após ajustes para os confundidores, a medida para a HAS foi atenuada (OR ajustada $=1,72$; IC95\%: 1,042,84), mas mantendo a significância estatística.

Considerando a expressão dessas medidas por estrato de idade, a HAS foi 2,15 vezes maior entre indivíduos com HAS e com pelo menos 54 anos de idade (OR bruta $=2,15$; IC95\%: 1,22-3,79) com significância estatística. Mesmo após ajuste, a associação se manteve (OR ajustada $=2,02$; IC95\%:1,07-3,83). Além disso, observa-se pela magnitude das medidas nos estratos, que a relação entre hábitos de vida e HAS foi mais forte no grupo com faixa etária mínima de 54 anos do que no de 18-53 anos.

\section{Discussão}

O principal achado deste estudo é que a prevalência de HA na população avaliada é maior do que os valores relatados em nível nacional. De acordo com o VIGITEL ${ }^{7}$, a prevalência de HA no conjunto das capitais brasileiras é $24,8 \%$. No presente estudo, a prevalência encontrada é de 67,2\%.

Estudos anteriores mostram que o sexo masculino pode apresentar maior concentração de fatores de risco para eventos cardiovasculares. ${ }^{10,12-14}$ Quanto ao hábito de fumar e diabetes, os achados mostraram que a associação só foi mantida nos não fumantes e não diabéticos. No caso da análise de subgrupo por diabetes, a medida de associação ajustada e por estrato de idade foi atenuada. Similarmente, outros estudos observaram o mesmo para esses subgrupos. ${ }^{15,16}$

Dados de outras investigações apontam que esses hábitos de vida têm mais chance de apresentar formas malignas da HA, que eleva a incidência de doenças cardiovasculares e ateroescleróticas e de morte súbita e que a restrição do etilismo é uma medida eficaz na redução da pressão arterial. ${ }^{17,18}$

A amostra estudada mostrou predominância de baixo consumo de álcool e tabaco, achados que convergem com os encontrados em alguns estudos. ${ }^{19-23}$ Todavia esse achado diverge do verificado nos resultados encontrados em outra investigação. ${ }^{24}$ Sugere-se que o fato da amostra do estudo ser, em maioria, composta de hipertensos, em acompanhamento, esse fato tenha 
corroborado para esse perfil de hábitos deletérios à saúde; Acredita-se que a redução da variabilidade da frequência cardíaca é um dos múltiplos mecanismos que leva o tabagista a desenvolver a hipertensão arterial. ${ }^{25,26}$

No entanto, a prevenção e o tratamento da HA também exige a necessidade da adoção de hábitos de vida saudável baseados numa alimentação balanceada, abandono de hábitos deletérios e realização de atividade física regular ${ }^{20,24}$. A prática regular de atividade física, por sua vez, combinada a um padrão alimentar adequado promove a redução da circunferência abdominal, e, consequentemente, a perda de peso, além de minimizar riscos cardiovasculares globais $^{21,27}$.

Estudo investigou os efeitos de um programa de atividade física na pressão arterial e a frequência cardíaca de 21 pacientes, sendo 6 destes, hipertensos. Os resultados acusam que houve redução da PAS e da frequência cardíaca de todos os pacientes após a prática de exercícios físicos. No tocante do grupo de hipertensos, este apresentou melhora significativa da PAS e queda da frequência cardíaca após a atividade física, quando comparados os valores no início do projeto. ${ }^{28}$

Desse modo, compreende-se que realizar exercícios físicos promove a redução da atividade simpática sobre o coração, e, consequentemente, redução da frequência cardíaca, assim como tem efeito hipotensor mais expressivo em indivíduos que iniciam a prática com valores de pressão arterial mais elevados, nesse caso, os hipertensos. Nesse sentido, vale salientar que, quando for incluída a utilização de medicamentos na terapêutica da HA, os mesmos devem acrescentar benefícios, e não substituir $o$ tratamento não farmacológico da atividade física. ${ }^{28,29}$

Embora não tenha sido observada significância estatística em alguns dos estratos das análises de subgrupos exploradas, a interpretação desses achados deve ser feita com cautela. A justificativa se deve, provavelmente, à falta de poder que impossibilita a detecção de diferenças existentes entre os indivíduos devido ao número reduzido de participantes em cada substrato.

Ressalta-se que a idade foi confirmada como modificadora de efeito na regressão logística e assim apresentada na descrição dos modelos de associação gerados. A literatura aponta que as frequências das doenças em estudo aumentam com a idade, ou seja, em adultos velhos ${ }^{15,19,20,24}$. Isso pode ser evidenciado pelas maiores médias de idade e frequências de aposentados nos hipertensos do presente estudo.

Outras variáveis como escolaridade, consulta ao dentista, colesterol HDL, obesidade e hemoglobina glicada foram incorporadas aos modelos finais a fim de se neutralizar os seus potenciais efeitos sobre a HAS, conforme discutido na literatura.

No que se refere aos fatores socioeconômicos como a escolaridade, é reconhecido amplamente a sua determinação na ocorrência dessa doença por repercutir na acessibilidade aos serviços de saúde e na apreensão das informações de saúde voltadas aos fatores de risco e o tratamento e controle da condição em estudo. ${ }^{6,19,30}$

Embora dados da VIGITEL evidenciem que a prevalência da HA é inversamente proporcional ao nível de escolaridade, neste estudo, a menor escolaridade foi detectada nos controles, grupo no qual houve maior frequência de pessoas que não trabalhavam. ${ }^{7}$ Esses dados levam a refletir que outros fatores socioeconômicos e demográficos, como a idade, estejam tendo maior impacto na distribuição da HA no perfil desta amostra.

Quanto às medidas bioquímicas e antropométricas incluídas, achados de estudos prévios mostram associação estatisticamente significante do colesterol HDL, obesidade e hemoglobina glicada 
(marcador de controle glicêmico) com a HA e eventos cardiovasculares. ${ }^{11,19,30}$

Algumas limitações são apontadas neste estudo como: valores de prevalência obtidos por estudo retrospectivo, o que não permite acompanhamento prospectivo a longo prazo; a não-representatividade da amostra, uma vez que foram incluídos apenas aqueles que estavam nos serviços de saúde e a forma de obtenção de algumas informações pode induzir a vieses de memória. Todavia, os achados da pesquisa vêm contribuir para ampliar as evidências cientificas sobre a influência dos hábitos de vida e HA.

Por fim, ressalta a necessidade da atuação da equipe multiprofissional na prática clínica para o tratamento integral do paciente, interrelacionando profissionais responsáveis a discutirem abordagens e condutas, garantindo qualidade $\mathrm{e}$ efetividade no atendimento, bem como estudos adicionais que proporcionem ainda mais subsídios para que as políticas de atenção e enfretamento desses agravos possam ser repensadas e reestruturadas.

\section{Conclusão}

Diante dos resultados, é possível concluir uma prevalência de HA, onde a maioria é do sexo feminino, tem faixa etária em maior índice superior a 54 anos de idade; vive com companheiros ou filhos; possuem maior índice de escolaridade; a renda familiar é maior que 3 salários mínimos, e tem as doenças cardiovasculares como a comorbidade principal.

É importante entender que os hábitos de vida dos sujeitos estão relacionados com o controle da HA. Desse modo, existe relação entre o cuidado da saúde e o hábito de vida. Por essa razão, tais informações reforçam a importância de ações que objetivem a melhora do perfil antropométrico e de pressão arterial na comunidade avaliada, com a premência de ações do poder público na promoção da saúde da comunidade estudada.

A aplicação da mudança dos hábitos de vida em indivíduos hipertensos tem efeito significativo e é indispensável, pois contribui com ações de intervenção efetivas na prevenção e tratamento do público atendido em unidades básicas de saúde.

\section{Referências}

1. Sociedade Brasileira de Cardiologia / Sociedade Brasileira de Hipertensão / Sociedade Brasileira de Nefrologia. VI Diretrizes Brasileiras de Hipertensão. Arq Bras Cardiol. 2010;95(1)1-51.

2. American Heart Association. Heart disease and stroke statistics - 2013 update: a report from the American Heart Association. Circulation. 2013; 127. Disponível em: https://doi.org/10.1161/CIR.0b013e31828124ad .

3. Oliveira TL, Miranda LP, Fernandes OS, Caldeira AP. Eficácia da educação em saúde no tratamento não medicamentoso da hipertensão arterial. Acta paul enferm. 2013; 26 (2): 179 -84. Disponível em: https://dx.doi.org/10.1590/S0103-21002013000200012

4. Rapsomaniki E, Timmis A, George J, Pujades-Rodriguez M, Shah AD, Denaxas S, et al. Blood pressure and incidence of twelve cardiovascular diseases: lifetime risks, healthy lifeyears lost, and age-specific associations in 125 million people. Lancet. 2014; 383 (9932): 1899-911. Disponível em: https://doi.org/10.1016/S0140-6736(14)60685-1

5. Mancia G, Fagard R, Narkiewicz K, Redón J, Zanchetti A, Böhm M, et al. ESH/ESC Guidelines for the management of arterial hypertension: the Task Force for the management of arterial hypertension of the European Society of Hypertension (ESH) and of the European Society of Cardiology (ESC). Am J Hypertens. 2013; 31 (28): 1281-357. Disponível em: https://doi.org/10.1093/eurheartj/eht151 
6. Sociedade Brasileira de Cardiologia. $7^{\text {a }}$ Diretriz Brasileira de Hipertensão Arterial. Arquivos Brasileiros de Cardiologia. Arq Bras Cardiol 2016; 107:1-83.

7. Ministério da Saúde, Brasil. Secretaria de Vigilância em Saúde. Departamento de Vigilância de Doenças e Agravos Não Transmissíveis e Promoção da Saúde. Vigitel 2015: vigilância de fatores de risco e proteção para doenças crônicas por inquérito telefônico. Brasília: Ministério da Saúde; 2017.

8. Ministério da Saúde, Brasil. SIAB - Sistema de Informação da Atenção Básica. 2017. Disponível em: http://www2.datasus.gov.br/SIAB/index.php?area=04.

9. Lima LM, Schwartz E, Muniz RM, Zillmer JGV, Ludtke I. Perfil dos usuários do Hiperdia de três unidades básicas de saúde do sul do Brasil. Rev Gaucha Enferm. 2011; 32(2):3239. Disponível em: http://dx.doi.org/10.1590/S1983-14472011000200016

10. Mendes TAB, Goldbaum M, Segri NJ, Barros MBA, César CLG, Carandina L. Factors associated with the prevalence of hypertension and control practices among elderly residents of São Paulo city, Brazil. Cad Saude Publica. 2013; 29(11):2275-86. Disponível em: http://dx.doi.org/10.1590/0102-311x00151312

11. Silveira J. Fatores associados à hipertensão arterial sistêmica e ao estado nutricional de hipertensos inscritos no programa Hiperdia. Cad Saude Colet. 2013; 21(2):129-34. Disponível em: http://dx.doi.org/10.1590/S1414-462X2013000200005

12. Darnaud C, Thomas F, Pannier B, Danchin N, Bouchard P. Oral Health and Blood Pressure: The IPC Cohort. Am J Hypertens. 2015; 28(10):1257-61. Disponível em: https://doi.org/10.1093/ajh/hpv025

13. Kawabata Y, Ekuni D, Miyai H, Kataoka K, Yamane M, Mizutani S, et al. Relationship between prehypertension/hypertension and periodontal disease: a prospective cohort study. Am J Hypertens. 2016;29(3): 388-96. Disponível em: https://doi.org/10.1093/ajh/hpv117

14. Ollikainen E, Saxlin T, Tervonen T, uominen AL, Knuuttila M, Jula A, et al. Association between periodontal condition and hypertension in a non-smoking population aged 30-49 years: results of the Health 2000 Survey in Finland. J Clin Periodontol. 2014; 41(12):1132-1138. Disponível em: https://doi.org/10.1111/jcpe.12316

15. Ahn YB, Shin MS, Byun JS, Kim HD. The association of hypertension with periodontitis is highlighted in female adults: results from the Fourth Korea National Health and Nutrition Examination Survey. J Clin Periodontol. 2015; 42 (11): 998-1005. Disponível em: https://doi.org/10.1111/jcpe.12471

16. Rivas-Tumanyan S, Campos M, Zevallos JC, Joshipura KJ. Periodontal disease, hypertension and blood pressure among older adults in Puerto Rico. J Clin Periodontol. 2013; 84(2):203-211. Disponível em: https://doi.org/10.1902/jop.2012.110748

17. Radovanovic CAT, Santos LA, Carvalho MDB, Marcon SS. Hipertensão arterial e outros fatores de risco associados às doenças cardiovasculares em adultos. Rev. Latino-Am. Enfermagem. 2014;22(4):547-53

18. Ikeda N, Inoue M, Isso H, Ikeda S, Satoh T, Noda M, et al. Adult mortality attributable to preventable risk factors for non-communicable diseases and injuries in Japan: a comparative risk assessment. PLoS Med. 2012;9(1):e1001160.

19. Carvalho SS, Silva TMA, Coelho JMF. Contribuições do tratamento não farmacológico para Diabetes Mellitus tipo 2. Rev Epidemiol Control Infect. 2015; 5(2):59-64. Disponível em: Disponível em: http://dx.doi.org/10.17058/reci.v5i2.5616

20. López NJ, Quintero A, Casanova PA, bieta CI, Baelum V, López R. Effects of periodontal therapy on systemic markers of inflammation in patients with metabolic syndrome: a controlled clinical trial. J Periodontol. 2012; 83(3):262-278. Disponível em: https://doi.org/10.1902/jop.2011.110227

21. Jahangiry L, Shojaeizadeh D, Montazeri A, Najafi M, Mohammad K, Abbasalizad Farhangi M. Modifiable lifestyle risk factors and metabolic syndrome: opportunities for a Web-Based Preventive Program. J Res Health Sci. 2014;14(4):303-307. 
22. Liu YL, Lu CW, Shi L, Liou YM, Lee LT, Huang KC. Low intensive lifestyle modification in young adults with metabolic syndorme a communit-based interventional study in Taiwan. Medicine (Baltimore). 2015; 94 (22): e916. Disponível em: https://10.1097/MD.0000000000000916

23. Rao DP, Dai S, Lagacé C, Krewski D. Metabolic syndrome and chronic disease. Chronic Dis Inj Can. 2014; 34 (1): 36-45.

24. Rao DP, Dai S, Lagacé C, Krewski D. Metabolic syndrome and chronic disease. Chronic Dis Inj Can. 2014; 34 (1): 36-45.

25. Gondim RM, Farah BQ, Santos CF, Ritti-Dias RM. O tabagismo e o fumo passivo estão relacionados com a variabilidade da frequência cardíaca em adolescentes homens? Einstein. 2015;13(1):27-33

26. Reza CG, Nogueira MS. O estilo de vida de pacientes hipertensos de um programa de exercício aeróbio: estudo na cidade de Toluca, México. Esc Anna Nery Rev Enferm. 2008; 12 (2): 265 - 70.

27. Iwashima Y, Kokubo Y, Ono T, Yoshimuta Y, Kida M, Kosaka T, et al. Additive interaction of oral health disorders on risk of hypertension in a japanese urban population: The Suita Study. Am J Hypertension. 2014; 27(5). Disponível em: https://doi.org/10.1093/ajh/hpt227

28. Damázio LCM, Filho GRS, Pereira ACL, Leão TLO, Ferreira LC, Mourão FN. Os efeitos de um programa de atividade física na pressão arterial e frequência cardíaca de pacientes hipertensos e deficientes físicos. Rev. Aten. Saúde. 2019;17(59):81-87. Disponível em: https://doi.org/10.13037/ras.vol17n59.5836

29. Miranda SS, Carvalho S, Andrade TM, Coelho JMF, Gomes-Filho IS. Atividade física e o controle glicêmico de pacientes com diabetes mellitus tipo II. Rev Bras Pesq Saúde. 2015; 17(3):33-40. Disponível em: http://periodicos.ufes.br/RBPS/article/view/14134/9963

30. Andrade SSA, Stopressão arterialSR, Brito AS, Chueri PS, Szwarcwald CL, Malta DC. Prevalência de hipertensão arterial autorreferida na população brasileira: análise da Pesquisa Nacional de Saúde, 2013. Epidemiol Serv Saúde. 2015; 24(2):297-304. Disponível em: https://doi.org/10.5123/S1679-49742015000200012

\section{Como citar este artigo:}

Carvalho SS, Oliveira BR. Hábitos e hipertensão: estudo realizado em hipertensos dos serviços públicos de saúde em Feira de Santana-BA. Rev. Aten. Saúde. 2020; 18(64): 22-31. 